\section{PROMOÇÃO DA SAÚdE NO CONTEXTO DAS REDES SOCIAIS SIGNIFICATIVAS}

\author{
HEALTH PROMOTION IN THE CONTEXT OF SIGNIFICANT SOCIAL NETWORKS
}

RESUMO: A promoção da saúde valoriza a atenção à integralidade e as relações sociais consideradas significativas. 0 presente texto objetivou discutir as relações entre a promoção da saúde e as redes sociais significativas. 0 conceito de promoção da saúde foi contextualizado por meio de políticas públicas, e a definição de redes sociais significativas foi complementada com a apresentação do instrumento de pesquisa e intervenção denominado de mapa de redes. Buscou-se apresentar pesquisas que relacionaram redes sociais significativas e saúde evidenciando as contribuições desse construto teórico no campo da Psicologia e saúde. Foram verificadas as aproximações conceituais entre promoção da saúde e as redes sociais, o que permite ressaltar a importância de ações articuladas considerando que os vínculos sociais representam fatores de proteção da saúde integral.

PALAVRAS-CHAVE: Promoção da Saúde; Redes Sociais Significativas; Psicologia Social.
ABSTRACT: Health promotion values integrated care, including the social relations considered significant. The aim of this work was to discuss the relationships between health promotion and significant social networks. The concept of health promotion was discussed in the context of public policies, and the definition of significant social networks was complemented by presenting a research and intervention instrument called network map.Studies associating significant social networks and health were presented, highlighting the contributions of this theoretical construct in the field of Psychology and Health.A conceptual proximity between health promotion and social networks was found, which emphasizes the importance of articulated actions, considering that social bonds represent protective factors for integrated health.

KEYWORDS: Health promotion; Significant social networks; Social Psychology.
ADRIANO VALÉRIO

DOS SANTOS

AZEVÊDO

MARCOS ANTÔNIO

DA SILVA

TOMÁS COLLODEL MAGALHÃES REIS

Universidade Tuiuti do Paraná (UTP),

Programa de PósGraduação em Psicologia, Curitiba/PR, Brasil

\title{
INTRODUÇÃO
}

Redes de apoio significativas representam "a soma de todas as pessoas que o indivíduo percebe ou sente como significativas do universo relacional no qual está inserido" (Sluzki, 1997, p.42). Estas pessoas auxiliam o indivíduo no enfrentamento de riscos e de vulnerabilidades e quando esses vínculos são saudáveis, sejam formais ou informais, repercutem na dimensão pessoal e coletiva do indivíduo e contribuem para o desenvolvimento de estratégias de enfrentamento de desafios. Essas interações sociais vivenciadas nas redes sociais significativas promovem a possibilidade de compartilhamento de experiências e de fortalecimento comunitário que contribuem para a qualidade de vida.

A promoção da saúde integra os níveis individual e coletivo (Buss, 2000), ao considerar que o nível individual consiste em atividades voltadas para a transformação dos comportamentos e estilos de vida no contexto familiar e comunitá- 
rio. Os objetivos destas atividades que estariam no controle dos indivíduos seriam educativas e relacionadas com os riscos passíveis de mudanças como, por exemplo, desenvolvimento de prática de atividade física e alimentação saudável. No segundo nível, as atividades da promoção da saúde estão voltadas para a coletividade e ao ambiente físico, social, econômico, político e cultural mais amplo e em que a saúde seria um produto de vários fatores que promoveriam a qualidade de vida através de um padrão adequado de alimentação, de habitação, de saneamento básico, de condições de trabalho, de acesso à educação.

Neste sentido, as redes sociais significativas promovem saúde no âmbito individual e coletivo. Percebe-se, dessa forma, que a saúde é produzida por meio das interações sociais. Este texto propõe apresentar as articulações entre promoção da saúde e redes sociais significativas. Na primeira parte do texto são discutidos os conceitos e a política pública de promoção da saúde, em seguida as noções de redes sociais significativas e resultados de pesquisas para refletir sobre as relações entre os aspectos sociais que fomentam a saúde integral.

\section{PROMOÇÃO DA SAÚDE}

A promoção da saúde supera a relação biomédica saúde/doença, pois envolve ações geradoras de qualidade de vida para indivíduos e coletivos quepossibilitam uma transformação das condições de vida, potencializando a saúde individual e coletiva, reduzindo as doenças decorrentes dos círculos sociais, econômicos e ambientais (Czresnia, 2009). O Ministério da Saúde compreende que a promoção da saúde representa um processo que in- tegra ações e estratégias que visam ao bem-estar individual e coletivo com o propósito de melhorar a qualidade de vida e saúde (Portaria n. 687, 2006). Essa compreensão precisa ser clara e distinta do conceito de prevenção para que isto possa facilitar a elaboração de práticas efetivas.

A prevenção exige uma ação antecipada fundamentada no conhecimento epidemiológico a fim de tornar improvável o progresso posterior da doença, controlando a transmissão de doenças infecciosas, reduzindo os riscos de doenças degenerativas ou outros agravos específicos por meio da divulgação de informações científicas que ajudam na mudança de hábitos da população (Czresnia, 2009). Já a Promoção da Saúde, segundo a autora, busca a transformação das condições de vida, do meio ambiente e do trabalho que implicará o fortalecimento da capacidade individual e coletiva para fazer escolhas que visam a aumentar a saúde e o bem-estar, demandando uma abordagem intersetorial.

Em um país continental como o Brasil, que é desigual social e economicamente, as pessoas em situação de pobreza constituem um grupo vulnerável para adoecimento. Vulnerabilidade social representa a falta ou impossibilidade de acesso a bens materiais, serviços de saúde, educação, trabalho e bem-estar que impossibilitam promover qualidade de vida (Ayres, França-Júnior, Calazans, \& Saletti-Filho, 1999). Para Abramovay (2002), a vulnerabilidade social ocorre quando há recursos insuficientes no grupo social que dificultam o enfrentamento das estruturas sociais que geram exclusão.

Para reduzir as desigualdades e as vulnerabilidades sociais, o governo federal - desde o início de 2000 - começou a discutir a construção de políticas 
públicas com o objetivo de promover avanços e rupturas com o modelo de saúde para que ocorram mudanças na qualidade de vida da população. Essa discussão avançou e, no início de 2006, o governo federal, através da Portaria MS/GM 687, de 30 de março, estabeleceu a Política Nacional de Promoção da Saúde [PNPS] com o seguinte objetivo:

Promover a qualidade de vida e reduzir vulnerabilidade e riscos à saúde relacionados aos seus determinantes e condicionantes - modo de viver, condições de trabalho, habitação, ambiente, educação, lazer, cultura, acesso a bens e serviços essenciais. (Portaria n. 687, 2006)

Na elaboração da PNPS de 2006 há um esforço em conceituar a promoção de saúde a partir de documentos internacionais que debateram a definição e as estratégias de um conceito de saúde amplo e transversal com estreita relação com a prevenção (Silva \& Baptista, 2015). A PNPS em 2010 foi atualizada para operar transversalmente, o que geraria redes de corresponsabilidades pela melhoria da qualidade de vida, levando em consideração a importância dos condicionantes e determinantes sociais da saúde na relação entre saúde-doença.

O documento da PNPS de 2006 foi redefinido em 2014, com a Portaria n. 2.446, de 11 de novembro, assinada pelo Ministro da Saúde Arthur Chioro, que justificou a mudança para propor novas ações de promoção da saúde em todo território nacional, garantindo que estejam em concordância com os princípios e diretrizes do SUS (Portaria n. 2446, 2014).

Art. $2^{\circ}$ A PNPS traz em sua base o conceito ampliado de saúde e o referencial teórico da promoção da saúde como um conjunto de estratégias e formas de produzir saúde, no âmbito individual e coletivo, caracterizando-se pela articulação e cooperação intra e intersetorial, pela formação da Rede de Atenção à Saúde (RAS), buscando articular suas ações com as demais redes de proteção social, com ampla participação e controle social.(Portaria n. 2446,2014 )

Entre os princípios da PNPS destaca-se a participação social que considera a visão de diferentes atores, agentes, grupos e comunidades para identificar os problemas e buscar uma intervenção em conjunto para as necessárias resoluções (Portaria n. 2446, 2014). Uma das diretrizes é apoiar e fomentar a educação permanente em promoção da saúde, ampliando o compromisso e gerando reflexão crítica dos gestores e trabalhadores da área, buscando desenvolver habilidades individuais e coletivas que venham fortalecer o desenvolvimento humano. Entre os eixos dos temas transversais está a vida no trabalho, que envolve a relação entre o trabalho formal e não formal nos setores primário, secundário e terciário da economia, levando em consideração a realidade urbana e rural, buscando oportunidades de estratégias para a promoção da saúde através de atividades desenvolvidas nestes espaços de maneira participativa e construtiva (Portaria n. 2446, 2014).

Os temas atuais prioritários da PNPS são: formação e educação permanente; alimentação adequada e saudável; práticas corporais e atividades físicas; enfrentamento do uso do tabaco e seus derivados; enfrentamento do uso abusivo de álcool e outras drogas; promoção da mobilidade segura, que visa à redução da mortalidade no trânsito por 
meio de educação e ações integradas e intersetoriais para promover a paz no trânsito; promoção da cultura da paz e dos direitos humanos; promoção do desenvolvimento sustentável (Portaria 2446, 2014). A PNPS representa um avanço importante na discussão da saúde e na contextualização de suas especificidades considerando as características dos brasileiros, o que permitiu ampliar o conceito de promoção da saúde valorizando os modos de viver, as relações sociais e a produção social da saúde na coletividade.

Segundo Silva e Baptista (2015), na redefinição da PNPS, em 2014, encontra-se em discussão de que maneira a promoção da saúde contribui de forma articulada para fomentar a qualidade de vida, as políticas de saúde e o desenvolvimento integral da sociedade brasileira. Neste sentido, um dos destaques refere-se à intrasetorialidade que busca construir, em meio à complexidade do setor da saúde, o debate da promoção da saúde nas ações promovidas pelo Sistema Único de Saúde (SUS). A PNPS de 2014 reconhece a trajetória da política do SUS, mas retoma algumas questões como, por exemplo, a discussão de risco à saúde decorrente dos determinantes sociais, econômicos, políticos, culturais e ambientais.

A PNPS permitiu provocar mudanças na maneira de organizar, planejar e avaliar o trabalho em saúde, e apresenta, em sua essência, a necessidade de estabelecer relações com as demais políticas públicas conquistadas pela população. A PNPS contribui para reduzir as desigualdades sociais e promover o desenvolvimento humano da população, pois se fundamenta nos princípios da integralidade, equidade, participação social e autonomia. Desta forma, espera-se que a população participe dos projetos de promoção da saúde com um olhar ampliado para o contexto so- cial reconhecendo o papel de cidadão brasileiro para a tomada de decisões.

A integralidade e a equidade representam os princípios doutrinários do SUS que fundamentam a Lei Orgânica da Saúde 8.080 (Lei n. 8080, 1990), mas estes princípios foram apresentados pela PNPS com novas definições. Na Lei Orgânica da Saúde, a integralidade propõe a articulação de ações e serviços preventivos e curativos para indivíduos e coletivos; a equidade refere-se à igualdade de assistência à saúde (Lei n. 8080, 1990). Na PNPS, a integralidade reconhece a complexidade, potencialidade e singularidade de indivíduos, grupos e coletivos para o desenvolvimento de intervenções por meio de trabalhos articulados e integrais; a equidade nas ações de promoção da saúde considera a distribuição igualitária a partir da análise de aspectos específicos de indivíduos e grupos (Portaria 2446, 2014). É possível verificar os avanços referentes às definições conceituais. Em síntese, a promoção da saúde é realizada mediante as necessidades de indivíduos e coletivos com o objetivo de desenvolver ações articuladas no setor saúde e nas instituições e comunidades que possam auxiliar o cuidado à saúde e o enfrentamento de situações.

Diante da desigualdade social no contexto brasileiro, existe o desafio de efetivar práticas que promovam a integralidade e equidade na assistência e nas estratégias de promoção da saúde (Buss, 2007). Portanto, as ações que objetivam promover saúde apresentarão efeitos positivos mediante a utilização adequada dos princípios que fundamentam a intervenção, para que isto possibilite a participação social visando à emancipação de indivíduos e coletivos.

Segundo Campos, Barros e Castros (2004), o Estado precisa assumir a responsabilidade de integrar a política da 
saúde com as demais políticas públicas sociais e econômicas para garantir que sejam implementadas e eficientes, e que isto possibilite que os agentes da saúde e a população sejam os representantes, os protagonistas de uma construção ampla de saúde. Os mesmos autores entendem que a mobilização dos setores da sociedade para promover a qualidade de vida fortalece os movimentos sociais, gerando autonomia do sujeito e do coletivo para que possam enfrentar as dificuldades por meio da construção de possibilidades que satisfaçam as necessidades da população. É nesse contexto que as redes sociais significativas cumprem um papel importante no desenvolvimento de ações que promovam saúde. É necessário, assim, considerar que os vínculos sociais são geradores de apoio e de fortalezas para a emancipação de indivíduos, grupos e coletivos.

\section{REDES SOCIAIS SIGNIFICATIVAS}

Redes sociais significativas são redes de apoio que contribuem para o fortalecimento da identidade da pessoa e do seu reconhecimento no grupo inserido. Representam, assim, um conjunto de pessoas que fornecem apoio para o indivíduo em determinados momentos da vida (Sluzki, 1997). Entende-se que quando as pessoas têm uma rede social que gera estabilidade, proteção e proporciona um ambiente confiável e participativo, estão menos propensas às doenças e têm maiores possibilidades de promover qualidade de vida.

Diante disto, é possível compreender que as redes sociais que promovem a saúde desenvolvem uma autoestima na pessoa que possibilita construir experiências capazes de promover o enfrentamento de suas lutas. Quando o sujeito é reconhecido pelo outro por meio da aceitação e valorização, consegue enfrentar o seu contexto de vida (Moré \& Crepaldi, 2012).

Neste sentido, Sluzki (1997) recomenda identificar a rede interpessoal que contribui para o fortalecimento da identidade da pessoa. Essa rede social do indivíduo é identificada através de um mapa mínimo que inclui todas as pessoas com quem a pessoa se relaciona (Figura 1). Segundo o autor, o mapa possui quatro quadrantes referentes aos seguintes vínculos: Família: Identifica-se como a família é composta, os vínculos significativos com os parentes, mesmo estando próximos geograficamente ou não; Amizades: As amizades podem compor uma parte importante da rede social significativa do sujeito, o que auxilia nos momentos difíceis e/ou quando os parentes não estão próximos; Relações de trabalho/escola: Muitas vezes essas são as únicas relações que o sujeito possui fora do contexto familiar; Relações comunitárias: identifica o pertencimento do sujeito nas relações com a comunidade (Sluzki, 1997).

Segundo Sluzki (1997), o mapa é representado por três círculos (interno, intermediário e externo), divididos em quatro quadrantes. $\mathrm{O}$ círculo interno engloba as relações mais próximas da pessoa; o círculo intermediário envolve as relações com menor grau de proximidade; já o círculo externo registra as relações esporádicas. Sluzki (1997) aponta que "o conjunto de habitantes desse mapa mínimo constitui a rede social pessoal do informante" (p.42). E para a aplicação do mapa de redes, apresenta-se o desenho do diagrama informando para o participante que o objetivo é mapear as pessoas que naquele momento estão fornecendo apoio. Para tanto, recomenda-se a construção de questões norteadoras para auxiliar a aplicação do mapa de redes (Moré \& Crepaldi, 2012). 


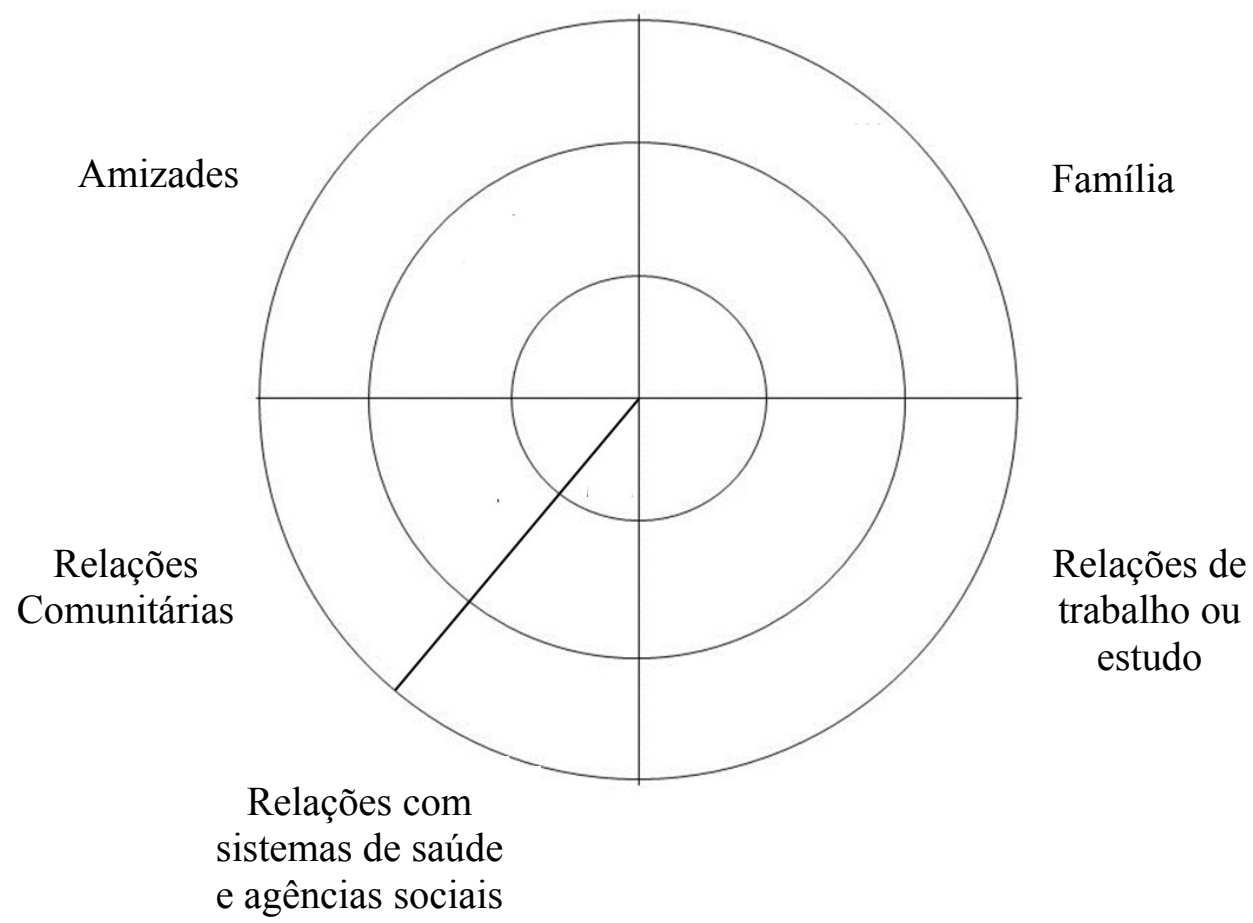

Fonte: Sluzki (1997)

O mapa identifica os relacionamentos significativos da pessoa e analisa a estrutura, funcionalidade e os atributos dos vínculos (Sluzki, 1997). A Tabela 1 apresenta os pontos que são analisados: (a) Estrutura da rede (tamanho, densidade, composição ou distribuição, dispersão, homogeneidade e heterogeneidade), (b) Funções da rede (companhia social, apoio emocional, guia cognitivo ou de conselhos, regulação social, ajuda material e de serviços, acesso a novos contatos), e (c) Atributos do vínculo (multidimensionalidade, reciprocidade, intensidade ou compromisso da relação, frequência de contatos, história da relação).
No que se refere à estrutura, redes de tamanho médio ( 8 a 10 pessoas) são mais eficientes que redes mínimas (Sluzki, 1997), e também redes com densidade média promovem eficácia. A partir disto, são analisadas as funções da rede que se referem aos tipos de apoio que são fornecidos e, em seguida, os atributos do vínculo que representam as especificidades do vínculo social. Trata-se de uma análise que considera a frequência referente ao número de pessoas na rede e aspectos qualitativos das funções e dos atributos do vínculo, por considerar que estas informações possibilitam contextualizar de que forma as redes foram construídas e de que maneira ocorre a dinâmica relacional. 


\section{Estrutura da rede}

- Tamanho: número de pessoas na rede.

- Densidade: conexão entre os membros da rede.

- Composição ou distribuição: distribuição das pessoas na rede.

- Dispersão: distância geográfica entre os membros da rede.

- Homogeneidade e heterogeneidade: identifica aspectos referentes a gênero (frequência de homens e mulheres, idade, cultura).

\section{Funções da rede}

- Companhia social: realização de atividades conjuntas.

- Apoio emocional: inclui empatia e compartilhamento de palavras de apoio.

- Guia cognitivo: pessoas que auxiliam o indivíduo por meio do esclarecimento de informações.

- Regulação social: pessoas que auxiliam o indivíduo na organização de papéis sociais e responsabilidades.

- Ajuda material: colaboração por meio de objetos e materiais.

- Acesso a novos contatos: abertura para novas redes.

\section{Atributos do vínculo}

- Função predominante do vínculo: caracteriza um tipo de vínculo.

- Multidimensionalidade: refere-se à quantidade de funções desempenhadas por uma pessoa.

- Reciprocidade: simetria entre duas pessoas.

- Intensidade ou compromisso da relação: grau de intimidade entre os membros da relação.

- Frequência de contatos: identifica de que maneira os vínculos são estabelecidos.

- História da relação: história do vínculo.

\section{REDES SOCIAIS SIGNIFICATIVAS E A PROMOÇÃO DA SAÚDE}

O conceito de redes sociais significativas tem fundamentado estudos no país que investigam como as relações da rede contribuem para o enfrentamento de situações de saúde e de vulnerabilidade psicossocial. Um dos estudos aborda as características do aleitamento materno e qual o papel rede nesse processo. Para tanto, os pesquisadores aplicaram o mapa de rede social em mulheres que estavam vivenciando o processo da amamentação e o bebê tinha menos de seis meses de idade (Souza, Souza,\& Tocantins, 2009). As redes sociais significativas destas mulheres eram formadas por pessoas da família e amigos (mãe, companheiro, sobrinho, vizinhas, irmãos, pais, primos, avós, tios, cunhada, sogra, madrinha da criança, patroas), no entanto, a maioria das mulheres teve pessoas da rede do gênero feminino. $\mathrm{O}$ ambiente familiar prevaleceu como o espaço prioritário de ajuda para o período pós-parto e no processo da amamentação, o que ajudou no compartilhamento de conhecimentos, experiências, hábitos e condutas nesse período. Embora as mulheres tenham 
mencionado que sua rede social era composta por familiares, amigos, colegas, vizinhos e profissionais de saúde, algumas destacaram que estavam sozinhas e que não tiveram o apoio da maioria dos profissionais nos serviços de saúde. Por outro lado, o apoio recebido pelos familiares contribuiu para a vivênciadesta experiência, pois o auxílio ajudou a promover a saúde na relação mãe-bebê nos cuidados maternos referentes à amamentação, configurando uma relevante relação entre o conceito de rede e promoção de saúde.

A pesquisa de Leonidas e Santos (2013) sobre transtornos alimentares consolida a compreensão do potencial do impacto positivo da rede em tratamentos de saúde. Esse estudo objetivou identificar as redes sociais significativas de mulheres com transtornos alimentares que frequentavam um hospital público com o objetivo de investigar como se configuram essas redes, o apoio social percebido e suas possíveis implicações na saúde. Identificou-se que as redes sociais apresentaram tamanhos médios, mas eram pouco diversificadas e com prevalência de pessoas da família, mesmo diante de conflitos e tensões. A figura materna representou a principal pessoa que fornece ajuda e foi possível identificar a multidimensionalidade, que se refere às diferentes funções desempenhadas, por exemplo, companhia social, guia cognitivo, ajuda material e regulação social. Nos outros quadrantes, amigos, profissionais da saúde e comunidade, verificou-se a existência de dificuldades no estabelecimento de vínculos devido à baixa frequência de contatos. O estudo apontou ainda a importância de o profissional da saúde identificar os vínculos sociais das pacientes, pelo fato de que este conhecimento pode oferecer subsídios para ampliar os recursos de tratamento, especialmen- te, para incluir as redes de apoio na condição de mediadores para prevenir novos agravos à saúde. Neste caso, as relações entre redes sociais e saúde apresentam relevância para o desenvolvimento da atenção integral às necessidades das pessoas numa perspectiva da integralidade.

O trabalho de Orlandi (2012) investigou como se dá o processo das redes sociais significativas de mulheres que vivem com o HIV no enfrentamento da soropositividade. Participaram do estudo mulheres infectadas pelo HIV que haviam sido diagnosticadas há mais de um ano, e identificou-se que as mulheres que enfrentaram o problema tinham redes sociais significativas eficientes e dinâmicas no que diz respeito à composição, às funções, à heterogeneidade, à frequência de contatos, à proximidade geográfica, à intimidade e às interconexões. Mesmo de maneira isolada, os profissionais de saúde tomavam iniciativas de acolher membros da rede social dessas mulheres no processo de enfrentamento da soropositividade, o que repercutiu positivamente na qualidade de ajuda. É possível destacar que a dinâmica das redes sociais destas mulheres contribui para o enfrentamento da situação, pois permite fortalecer os vínculos e minimizar riscos referentes ao agravamento da doença crônica, aspectos que indicam a promoção da saúde.

Ainda sobre a temática de HIV, na pesquisa de Zuben, Rissi e Guanaes-Lorenzi (2013) foi apresentado um estudo de caso sobre uma mulher portadora de HIV/aids para compreender como se configura a sua rede social significativa e como isto influencia na saúde. Os resultados permitiram identificar uma rede de tamanho médio com pessoas da família e amigos que fornecem apoio emocional e companhia social, pois, segundo as autoras, 
isto possibilita a promoção da saúde, o que foi fundamental para garantir qualidade de vida e desenvolver recursos para o enfrentamento das dificuldades advindas do diagnóstico de HIV/aids.

O trabalho de Azevêdo (2016) procurou identificar as redes sociais significativas de familiares cuidadores de crianças hospitalizadas com queimaduras. Identificou-se que a rede social significativa foi constituída pelas pessoas da equipe de saúde e da família. $\mathrm{O}$ apoio emocional e o suporte social possibilitaram fortalecer a capacidade de enfrentar as situações de ansiedade no contexto hospitalar. $\mathrm{Na}$ pesquisa de Menezes, Moré e Barros (2016), na qual foram analisadas as redes sociais de cuidadores de crianças hospitalizadas na pediatria, foram encontrados resultados semelhantes, pois a família e a equipe de saúde representaram as principais redes, cujas funções se referem ao apoio emocional, ajuda material, espiritual e guia cognitivo. Em relação a estas pesquisas, é importante ressaltar o papel das redes sociais na situação de doença e hospitalização, pois o enfrentamento da ansiedade e o desenvolvimento de interações sociais representam formas que promovem saúde. Estes aspectos geradores de saúde possibilitam minimizar o sofrimento vivenciado pelos cuidadores de crianças hospitalizadas, pois o cuidador necessita de apoio social para auxiliar a criança.

No estudo de Krenkel, Motta e Moré (2015) procurou-se compreender a dinâmica relacional das redes significativas de mulheres que sofreram violência no contexto familiar e foram acolhidas em casa-abrigo, uma vez que as redes sociais e o suporte institucional configuram ferramentas estratégicas para o enfrentamento desse fenômeno. As redes sociais sig- nificativas no contexto de violência demonstraram a importância da intensidade de suas relações mútuas, nas quais a ajuda foi oferecida através de apoio emocional e material. O estudo dos quadrantes apontou a presença de uma rede social significativa pequena e dispersa, o que denota um isolamento social dessa mulher que, antes de ir para o abrigo, ficou exposta por um período longo à violência. Algumas situações resultaram no isolamento das participantes de sua rede relacional: (a) a pessoa que cometeu a violência demonstra calma e bondade na frente dos familiares; (b) por conta do medo e da vergonha, as mulheres não contam à família e aos amigos os detalhes da violência; (c) pela existência de um consenso familiar de que brigas entre parceiros íntimos são normais e que devem ser resolvidas entre eles. Algumas participantes relataram que não falaram sobre a situação de violência para amigos e demais parentes porque os seus parceiros não permitiram que elas comentassem sobre isso, sendo algumas ameaçadas de morte. Esta situação levou ao distanciamento e ao isolamento de mulheres violentadas de suas redes sociais significativas. Os resultados desta pesquisa devem ajudar no trabalho/acolhimento dos profissionais da saúde e das equipes multidisciplinares para que elaborem estratégias de proteção às mulheres, oportunizando a superação da situação de violência. $\mathrm{O}$ isolamento social identificado neste estudo indica possíveis repercussões para o adoecimento destas mulheres; isto demonstra que a falta de apoio social representa um elemento que dificulta o enfrentamento de situações, o que compromete a saúde e aspectos da qualidade de vida.

Nestas pesquisas foi possível identificar como as redes sociais significativas exercem um papel relevante no 
enfrentamento de situações e repercutem na saúde integral. Isto mostra que as fontes de apoio social potencializam a capacidade do indivíduo de vivenciar as experiências, principalmente nas situações de adversidades. Os resultados das pesquisas apresentadas possibilitam a construção da relação entre rede social significativa e promoção de saúde, mesmo que esse tema não tenha sido objeto direto de investigação, com exceção da pesquisa de Zuben et al. (2013), na medida em que se compreende que as dimensões da rede são promotoras de cuidado em diferentes âmbitos da vida do indivíduo e que impactam diretamente na saúde geral. Para Sluzki (1997), a qualidade da rede de relações constitui-se num elemento observável em várias faces da vida do indivíduo, que se configura, dessa forma, como fatores determinantes de saúde.

O mapa de redes representa um instrumento que viabiliza o desenvolvimento de intervenções, pois quando os fatores de proteção de uma rede são identificados, é possível promover fortalecimentos para a sua manutenção. Por outro lado, diante de fatores de risco, baixa concentração de pessoas na rede e falta de apoio, é possível contextualizar estes aspectos entre os participantes e procurar recursos para a construção de uma rede social geradora de saúde.

\section{CONSIDERAÇÕES FINAIS}

As redes sociais significativas auxiliam o indivíduo no enfrentamento das situações e representam fontes de promoção da saúde. O entendimento ampliado de promoção da saúde permite que as relações sociais e as interações desenvolvidas sejam entendidas enquanto forma de ajudar o indivíduo em determinadas situações. A utilização da pesquisa por meio de mapa de redes permite refletir de maneira crítica sobre intervenções psicossociais que promovam saúde em diferentes contextos.

O conceito de rede social significativa constitui-se em um instrumental teórico e de intervenção que contribui para o desenvolvimento de ações em Promoção de Saúde na medida em que possibilita compreender como a rede de relações sociais impacta o desenvolvimento social e psicológico do indivíduo bem como o seu comportamento frente a demandas de atenção em saúde e de vulnerabilidade social. O uso desse construto teórico possibilita, portanto, a consolidação de ações promotoras de saúde, na perspectiva da integralidade e reconhecimento do impacto do contexto social na experiência de saúde e doença.

Cabe ressaltar a potência do mapa de redes como um instrumento de pesquisa e intervenção que possibilita a visualização da configuração das relações sociais facultando a avaliação psicossocial do indivíduo e/ou demanda em situação. Dessa forma, o desenho das relações evidenciado no mapa pode fomentar ações de saúde que valorizem a integralidade que integra a perspectiva de promoção de saúde.

\section{REFERÊNCIAS}

Abramovay, M. (2002). Juventude, violência e vulnerabilidade social na América Latina: desafios para políticas públicas. Brasília, DF: UNESCO. Ayres, J. R. C. M., França-Júnior, I., Calazans, G. J., \& Saletti-Filho, H. C. (1999). Vulnerabilidade e prevenção em tempos de Aids. In R. M. Barbosa\& R. G. Parker (Orgs.), Sexualidades pelo avesso: direitos, 
identidade e poder (pp. 49-72). São

Paulo: Editora 34.

Azevêdo, A.V. S. (2016). Ansiedade, enfrentamento e redes sociais significativas de familiares cuidadores de crianças com queimaduras. Tese de Doutorado, Programa de Pós-graduação emPsicologia, Universidade Federal de Santa Catarina, Florianópolis, SC.

Buss, P.M. (2000). Promoção da saúde e qualidade de vida. Revista Ciência e Saúde Coletiva, 5(1), 163-177. doi: 10.1590/ S1413-81232000000100014

Campos, G.W., Barros, R.B., \& Castro, A.M., (2004). Avaliação de política nacional de promoção da saúde. Ciência \& Saúde Coletiva, 9(3), 745-749. doi: 10.1590/ S1413-81232004000300025

Czeresnia, D. (2009). O conceito de saúde e a diferença entre prevenção e promoção. In D. Czeresnia \& C. M. Freitas (Orgs.), Promoção da saúde: conceitos, reflexões, tendências (pp. 43-57). Rio de Janeiro: Fiocruz.

Krenkel, S.y Motta, C. C. L.s \& Moré, C. L. 0. 0. (2015). As redes sociais significativas de mulheres acolhidas em casa-abrigo. Paidéia, 25(60), 125-133. doi: 10.1590/1982-43272560201515

Lei n.8.080, de 19 de setembro de 1990 . (1990).Sistema Único de Saúde. (SUS). Lei Orgânica da Saúde. Brasília, DF: Presidência da República/ Casa Civil. Recuperado de http:// www.planalto.gov.br/ccivil_03/leis/ L8080.htm

Leonidas, C. \& Santos, M. A. (2013). Redes Sociais significativas de mulheres com transtornos alimentares. Psicologia Reflexão \& Crítica, 26(3), 561-571. doi: 10.1590/ S0102-79722013000300016

Menezes, M.s Moré, C.L.0.0., \& BarroS, L. (2016). As redes sociais dos familiares acompanhantes durante inter- nação hospitalar de Crianças. Revista da Escola de Enfermagem da USP, 50(n.spe), 107-113. doi: 10.1590/ S0080-623420160000300016

Moré, C.L.0. O. \& Crepaldi, M.A. (2012). O Mapa de Rede Social significativa como instrumento de investigação no contexto da pesquisa qualitativa. Nova Perspectiva Sistêmica,21(43), 84-98. http://www.revistanps.com. br/index.php/nps/article/view/265

Orlandi, R. (2012). Participação da rede social significativa de mulheres que vivem e convivem com o HIV no enfrentamento da soropositividade. Tese de Doutorado, Programa de Pós-graduação em Psicologia, Universidade Federal de Santa Catarina, Florianópolis, SC.

Portaria n. 687, de 30 de março de 2006. (2006). Aprova a Política Nacional de Promoção da Saúde [PNPS].Brasília, DF: Ministério da Saúde.

Portaria n. 2.446, de 11 de Novembro de 2014. (2014). Redefine a Política Nacional de Promoção da Saúde [PNPS]. Brasília, DF:Ministério da Saúde. Recuperado de http:// bvsms.saude.gov.br/bvs/saudelegis/ gm/2014/prt2446 11 11-2014.html Silva, P.F. A.\& Baptista, T.W.F. (2015). A Política Nacional de Promoção da Saúde: texto e contexto de uma política. Saúde em Debate, 39(n.spe), 91-104. doi: 10.5935/0103-1104.2015S005327

Sluzki, C.E. (1997). A Rede Social na Prática Sistêmica: Alternativas Terapêuticas. São Paulo: Casa do Psicólogo.

Souza, M.H.N., Souza, I.E.O., \& Tocantins, FR. (2009). A utilização de referencial metodológico de rede social na assistência de enfermagem a mulheres que amamentam. Revista Latino-Americana de Enfermagem, 17(3), 354-360. doi:10.1590/ $\underline{\text { S0104-11692009000300012 }}$ 
Zuben, J.V.V., Rissil, M.R.R., \& Guanaes-Lorenzi, C. (2013). A rede social significativa de uma mulher após o diagnóstico de HIV/ AIDS. Psicologia em Estudo, 18(2), 211-221. doi: 10.1590/ S1413-73722013000200003

\section{ADRIANO VALÉRIO DOS SANTOS} AZEVÊDO

E-mail: adrianoazevedopsi@yahoo.com

\section{MARCOS ANTÔNIO DA SILVA}

E-mail: pr.marcosansi@gmail.com

TOMÁS COLLODEL MAGALHÃES REIS

E-mail: tomreis@gmail.com 